\title{
A VISCOSITY SOLUTION OF THE HAMILTON - JACOBI EQUATION WITH EXPONENTIAL DEPENDENCE OF HAMILTONIAN ON THE MOMENTUM
}

\author{
Lyubov Shagalova \\ N.N. Krasovskii Institute of Mathematics and Mechanics \\ Ural Branch of the Russian Academy of Sciences \\ Yekaterinburg, Russia \\ shag@imm.uran.ru
}

Article history:

Received 31.10.2021, Accepted 25.11.2021

\begin{abstract}
The initial - boundary value problem is considered for the Hamilton-Jacobi of evolutionary type in the case when the state space is one-dimensional. The Hamiltonian depends on the state and momentum variables, and the dependence on the momentum variable is exponential. The problem is considered on fixed bounded time interval, and the state variable changes from a given fixed value to infinity. The initial and boundary functions are subdifferentiable. It is proved that such a problem has a continuous generalized (viscosity) solution. The representative formula is given for this solution. Sufficient conditions are indicated under which the generalized solution is unique. Hamilton-Jacobi equations with an exponential dependence on the momentum variable are atypical for theory, but such equations arise in practical problems, for example, in molecular genetics.
\end{abstract}

\section{Key words}

Hamilton-Jacobi equation, initial-boundary value problem, viscosity solution, characteristics, subdifferentials

\section{Introduction}

Some practical problems and applied research, in particular, in molecular genetics [Saakian, Rozanova and Akmetzhanov, 2008], lead to the need to study Hamilton - Jacobi equations with an exponential dependence of the Hamiltonian on the momentum (impulse) variable. Such equations are atypical for the theory of Hamilton Jacobi equations, so there are no general existence and uniqueness theorems for solutions of equations of this type. Moreover, such equations, as a rule, do not have classical solutions, and the solution must be understood in some generalized sense.

This paper relies on the concept of a viscosity/minimax approach [Crandall and Lions, 1983; Subbotin, 1995] to defining a solution. This concept of solution is used in various applications of partial differential equations, in particular, in optimal control and differential games. However, exact formulas for solutions can be obtained in very few special cases. Therefore, a lot of research is aimed at developing methods for the numerical construction of solutions (see, for example, [Kolpakova and Tokmantsev, 2012; Filippova, 2013]).

An initial-boundary value problem for the HamiltonJacobi equation of the evolutionary type with a onedimensional state vector is considered in this paper. The initial and boundary function are supposed to be subdifferentiable and such that their Dini derivatives are finite. A procedure to construct a continuous viscosity solution is proposed, and a representative formula for this solution is obtained. Constructions are based on solving variational problems with moving boundaries and on the method of generalized characteristics [Subbotina, 2006]. Also, sufficient conditions are indicated under which such a solution is unique.

The problem considered here, which is of independent interest, arises when constructing a continuous viscous solution to an initial problem with a discontinuous threecomponent Hamiltonian [Shagalova, 2021]. Earlier, the Cauchy problem for an equation of such a type with a concave Hamiltonian was studied [Subbotina and Shagalova, 2016] in a domain bounded in the state variable.

\section{Problem Statement}

Let time moment $T>0$ and value $x^{*} \in R$ be given. In the region $G^{+}=\left\{(t, x) \mid 0<t<T, x>x^{*}\right\}$ the 
following Hamilton-Jacobi equation is considered

$$
\frac{\partial u}{\partial t}+H\left(x, \frac{\partial u}{\partial x}\right)=0
$$

Hamiltonian has the form

$$
H(x, p)=f(x) e^{p} .
$$

Here $f(\cdot)$ is continuously differentiable non-decreasing function such that

$$
0<f\left(x^{*}\right) \leq f(x)<K, \quad x \in\left(x^{*}, \infty\right),
$$

where $K$ is a given positive number. Continuous functions $u_{0}: R \rightarrow R$ and $\varphi:[0, T] \rightarrow R$ are also given such that

$$
\begin{gathered}
D_{-} u_{0}(x) \neq \emptyset \quad \forall x \in R \\
D_{-} \varphi(t) \neq \emptyset \quad \forall t \in(0, T) .
\end{gathered}
$$

Here $D_{-} \varphi(t)$ denotes the subdifferential of function $\varphi$ at the point $t$.

It is supposed that the left derivative $u_{0-}^{\prime}(0)$ of function $u_{0}$ at point $x^{*}$ and the left derivative $\varphi_{-}^{\prime}(0)$ of function $\varphi$ at point 0 exist, and the following equalities hold

$$
\varphi(0)=u_{0}\left(x^{*}\right), \quad \varphi_{-}^{\prime}(0)=u_{0-}^{\prime}\left(x^{*}\right) .
$$

We also assume that all Dini derivatives of functions $u_{0}$ and $\varphi$ are finite at all points $x \in R$ and at all points $t \in(0, T)$, respectively.

It is required to construct a viscosity solution [Crandall and Lions, 1983] $u(\cdot, \cdot)$ of the equation (1) which is continuous on the set $\mathrm{cl}^{+}$- the closure of the domain $G^{+}$, and such that the following initial and boundary conditions are satisfied

$$
\begin{gathered}
u(0, x)=u_{0}(x), \quad x \in R, x \geq x^{*} \\
u\left(t, x^{*}\right)=\varphi(t), \quad t \in[0 ; T] .
\end{gathered}
$$

\section{Subdifferentials and Viscosity Solutions}

Let us recall some known concepts of nonsmooth analysis that we use in the paper.

Let a set $G \subset R^{n}$ be given. The symbol $C(G)$ denotes the set of functions that are continuous on $G$. For vectors $\xi \in R^{n}$ and $h \in R^{n}$ their inner product will be denoted as $\langle\xi, h\rangle$. The norm of vector $h$ will be denoted as $|h|$.

Let $g(\cdot) \in C(G)$, and $y \in G$.

The subdifferential of function $g$ at point $y$ is the set $D^{-} g(y)=$

$$
\left\{\xi \in R^{n} \mid \liminf _{h \rightarrow 0} \frac{g(y+h)-g(y)-\langle\xi, h\rangle}{|h|} \geq 0\right\} .
$$

The superdifferential of function $g$ at point $y$ is the set $D^{+} g(y)=$

$$
\left\{\xi \in R^{n} \mid \limsup _{h \rightarrow 0} \frac{g(y+h)-g(y)-\langle\xi, h\rangle}{|h|} \leq 0\right\} .
$$

Remark. It should be noted that the sub-/super- differentials defined above are commonly known as Fréchet sub/super- differentials (see, for example, [Kruger, 2003]).

It is known that a continuous function $g(\cdot)$ is (Fréchet) differentiable at $y \in G$ if and only if $D^{-} g(y) \cap$ $D^{+} g(y) \neq \emptyset$. Moreover, in this case, $D^{-} g(y)=$ $D^{+} g(y)=D g(y)$, where $D g(y)$ denotes the Fréchet derivative of $g$ at point $y$.

Definition 1. Continuous function $g(\cdot)$ will be called subdifferentiable if at each interior point of its domain $G$ its subdifferential is not empty:

$$
D^{-} g(y) \neq \emptyset, \forall y \in \operatorname{int} G .
$$

For any upper continuous function the set of points at which it is superdifferentiable is dense [Ferrera, 2014, Theorem 4.21]. Any subdifferentiable function is continuous, so the set of its differentiability points is dense too.

Let $\psi(\cdot)$ be a continuous function of one variable, $\psi$ : $I \rightarrow R$, where $I$ is an interval, $I \subseteq R$. Then, for any $y \in I$, four Dini derivatives are defined. The lower left and upper left Dini derivatives are defined respectively as

$$
\begin{aligned}
& \psi_{-}(y)=\liminf _{h \rightarrow-0} \frac{\psi(y+h)-\psi(y)}{h}, \\
& \psi^{-}(y)=\limsup _{h \rightarrow-0} \frac{\psi(y+h)-\psi(y)}{h} .
\end{aligned}
$$

The lower right and upper right Dini derivatives are defined similarly.

$$
\begin{aligned}
& \psi_{+}(y)=\liminf _{h \rightarrow+0} \frac{\psi(y+h)-\psi(y)}{h}, \\
& \psi^{+}(y)=\limsup _{h \rightarrow+0} \frac{\psi(y+h)-\psi(y)}{h} .
\end{aligned}
$$

The following statement is valid.

Lemma 1. Let $\psi(\cdot)$ be a subdifferential function of one variable, $\psi: I \rightarrow R$, where $I$ is an interval, $I \subseteq R$. If for all $y \in I$ all Dini derivatives $\psi_{-}(y), \psi^{-}(y), \psi_{+}(y)$, $\psi^{+}(y)$ are finite numbers, then subdifferential $D \psi^{-}(y)$ is bounded for all $y \in I$ and $\psi(\cdot)$ is differentiable almost everywhere on $I$.

Proof. It follows from the definitions of the subdifferential and the Dini derivatives that the subdifferential is closed interval in $\mathrm{R}$.

$$
D \psi^{-}(y)=\left[\psi^{-}(y), \psi_{+}(y)\right], \quad y \in I .
$$

Since Dini derivatives are finite numbers, we obtain from (9) that the subdifferential is a bounded segment. 
By Denjoy-Young-Saks theorem [Bruckner, 1994, Theorem 4.4], with the possible exception of a null set, interval $I$ on which continuous function $\psi(\cdot)$ is defined can be decomposed into four sets:

$A_{1}$, on which $\psi(\cdot)$ has a finite derivative;

$A_{2}$, on which $\psi_{-}(y)=-\infty, \quad \psi^{+}(y)=+\infty$, $\psi^{-}(y)=\psi_{+}(y) \neq \pm \infty$

$A_{3}$, on which $\psi^{-}(y)=+\infty, \quad \psi_{+}(y)=-\infty$, $\psi_{-}(y)=\psi^{+}(y) \neq \pm \infty$;

$A_{4}$, on which $\psi_{-}(y)=\psi_{+}(y)=-\infty$, $\psi^{-}(y)=\psi^{+}(y)=+\infty$.

Since the Dini derivatives are finite, the sets $A_{2}, A_{3}$ and $A_{4}$ are empty. So, function $\psi(\cdot)$ is differentiable almost everywhere on $I$.

The viscosity solution of the equation (1) can be determined in many ways different in form, but essentially equivalent. Here it is more convenient for us to define such a solution using the differential inequalities for suband superdifferentials.

Definition 2. Viscosity solution of equation (1) is a continuous function $u(t, x):[0, T] \times\left[x^{*}, \infty\right) \rightarrow R$ such that the following inequalities are valid

$$
\begin{gathered}
a+H(x, s) \leq 0, \\
\forall(t, x) \in(0, T) \times\left(x^{*}, \infty\right), \quad(a, s) \in D^{+} u(t, x), \\
a+H(x, s) \geq 0, \\
\forall(t, x) \in(0, T) \times\left(x^{*}, \infty\right), \quad(a, s) \in D^{-} u(t, x) .
\end{gathered}
$$

\section{Construction of the Solution. Main Result}

The Hamiltonian has the form (2), where $f(x)>0$. So, the Hamiltonian $H$ is convex with respect to momentum variable $p$. Consider the conjugate to Hamiltonian function $H^{*}$, which is defined as

$$
H^{*}(x, q)=\sup _{p \in R}\{p q-H(x, p)\} .
$$

It is easy to see that for the Hamiltonian (2) the conjugate function has the following form.

$$
H^{*}(x, q)= \begin{cases}+\infty, & \text { if } q<0 \\ 0, & \text { if } q=0 \\ q \ln \left(\frac{q}{f(x)}\right)-q, & \text { if } q>0\end{cases}
$$

The characteristic system for equation (1) with Hamiltonian (2) has the form

$$
\begin{gathered}
\dot{x}=H_{p}(x, p)=f(x) e^{p}=H(x, p), \\
\dot{p}=-H_{x}(x, p)=-f^{\prime}(x) e^{p}, \\
\dot{u}=p H_{p}(x, p)-H(x, p)=(p-1) H(x, p) .
\end{gathered}
$$

Here symbols $H_{x}$ and $H_{p}$ denote derivatives with respect to the variables $x$ and $p$, respectively.

The initial manifold from which it is necessary to release characteristics is split into two parts.

$$
\begin{gathered}
\left\{(t, x, z) \mid t=0, x \geq x_{*}, z=u_{0}(x)\right\} \cup \\
\left\{(t, x, z) \mid 0 \leq t<T, x=x^{*}, z=\varphi(t)=u\left(t, x^{*}\right)\right\},
\end{gathered}
$$

so one must consider system (12) with two sets of initial conditions.

I. For characteristics starting at the moment $t=0$ initial conditions are of the next form

$$
x(0, y)=y, p(0, y) \in D^{-} u_{0}(y), z(0, y)=u_{0}(y),
$$

where $y \in\left[x^{*} ; \infty\right)$. If initial function $u_{0}$ is nondifferentiable at point $y$ then the whole bunch of characteristics starts from point $(0 ; y)$. It follows from Lemma 1 that on the line $t=0$ the set of such points of non-differentiability has measure zero.

II. For characteristics starting at the moment $t=\tau$, $\tau \in(0, T)$ from points on line $x=x^{*}$ initial conditions have the form

$$
x(\tau)=x^{*}, \quad p(\tau) \in D^{-} \varphi(\tau), \quad z(\tau)=\varphi(\tau) .
$$

Here $\varphi(\tau)=u\left(\tau, x^{*}\right)$. If $\varphi$ is non-differentiable at the point $\tau$ then the whole bunch of characteristics starts from point $\left(\tau ; x^{*}\right)$. It follows from Lemma 1 that the set of such points of non-differentiability has measure zero.

It is not difficult to receive from the first two equations of system (12) that $p=-\ln C f(x)$, and state components of characteristics are straight lines of the form $x=x_{0}+f\left(x_{0}\right) e^{p_{0}} t$. Note that the slopes $f\left(x_{0}\right) e^{p_{0}}$ of all these lines are positive.

Let

$$
\begin{aligned}
& \Gamma_{+}=\left\{(t, x) \mid t=0, x \geq x^{*}\right\} \cup \\
&\left\{(t, x) \mid 0 \leq t<T, x=x^{*}\right\} .
\end{aligned}
$$

For point $(\bar{t}, \bar{x}) \in G_{+}$define $X\left(\Gamma_{+} ; \bar{t}, \bar{x}\right)$ as the set of all state characteristics $x(\cdot)$ starting from $\Gamma_{+}$such that the condition $x(\bar{t})=\bar{x}$ is satisfied. One can check that the set $X\left(\Gamma_{+} ; \bar{t}, \bar{x}\right)$ is not empty.

Now, relying on the method of generalized characteristics, one can write down the following representative formula for solution of the initial-boundary value problem (1)-(8). $u(t, x)=$

$$
\begin{gathered}
\min _{X\left(\Gamma_{+} ; t, x\right)}\left\{u\left(\tau^{\natural}, x^{\natural}\right)+\int_{\tau^{\natural}}^{t}\left(p(\tau) H_{p}(x(\tau), p(\tau))\right.\right. \\
-H(x(\tau), p(\tau))) d \tau\} .
\end{gathered}
$$

Here $\left(\tau^{\natural}, x^{\natural}\right)$ - point in $\Gamma_{+}$from which the state characteristic $x(\cdot)$ starts; $p(\cdot)$ - the corresponding impulse (momentum) characteristic,

$$
u\left(\tau^{\natural}, x^{\natural}\right)=\left\{\begin{array}{l}
u_{0}\left(x^{\natural}\right), \text { if } \tau^{\natural}=0 \\
\varphi\left(\tau^{\natural}\right), \quad \text { if } 0 \leq \tau^{\natural}<T, x^{\natural}=x^{*} .
\end{array}\right.
$$

Note that the minimum in the expression (15) is attained.

So, now one can formulate the main result of this paper as the following statement. 
Theorem 1. There exists a continuous viscosity solution $u:[0, T] \times\left[x^{*}, \infty\right) \rightarrow R$ to initial - boundary problem (1)-(8).

Proof. It is not difficult to see that function $u$ defined by representative formula (15) is continuous on $[0, T] \times\left[x^{*}, \infty\right)$ and satisfies initial condition (7) and boundary condition (8). By construction, this function satisfies differential inequalities (10), (11). So, function (15) is a continuous viscosity solution to initial - boundary problem (1)-(8).

\section{On Uniqueness of the Solution}

In the general case, it cannot be argued that the solution to the initial-boundary problem (1)-(8) is unique. Below in this section we give two statements containing sufficient conditions under which the solution is unique.

Theorem 2. Let $M>x^{*}$. In the bounded region

$$
G_{+}^{M}=\left\{(t, x) \mid 0 \leq t \leq T, x^{*} \leq x<M\right\}
$$

the solution to initial-boundary Problem (1)-(8) is unique.

Proof of Theorem 2 is based on the fact that in the bounded region $G_{+}^{M}$ the momentum variable $p$ is also bounded, and Hamiltonian $H$ is Lipschitz continuous, so the known [Subbotin, 1995] uniqueness conditions for the minimax / viscosity solution are satisfied.

Theorem 3. Let $f(\cdot)$ be a constant function, $f(x)=$ $f\left(x^{*}\right), \forall x \geq x^{*}$, and function $u_{0}$ satisfy the global Lipschitz condition

$\left|u_{0}\left(x_{1}\right)-u_{0}\left(x_{2}\right)\right| \leq L\left|x_{1}-x_{2}\right|, \quad x_{1} \in R, \quad x_{2} \in R$.

Then the solution to initial-boundary Problem (1)-(8) is unique.

Proof of Theorem 3 is based on using known [Bardi and Evans, 1984] Hopf formula for Hamilton -Jacobi equation with convex Hamiltonian.

The solution, the construction of which is described in the previous section, in the domain $G^{+}$is formed from the characteristics - solutions of the system of ordinary differential equations (12). In this case, two types of characteristics are considered: the first start at the moment $t=0$ with the initial conditions (13), and the second start at the moments $\tau \in[0, T)$ with the initial conditions (14).

Characteristics of the second type are involved in the formation of a solution only in a bounded region. There exists number $K>x^{*}$ such that in the region $G^{+} \backslash c l G_{+}^{K}$ the solution is constructed only from characteristics of the first type, and in this domain the solution of the problem (1)-(8) coincides with the solution of the Cauchy problem for the following Hamilton-Jacobi equation

$$
\frac{\partial u}{\partial t}+f\left(x^{*}\right) e^{\partial u / \partial x}=0, \quad t \in(0, T), x \in R
$$

with initial condition

$$
u(0, x)=u_{0}(x), \quad x \in R
$$

It follows from [Bardi and Evans, 1984, Theorem 2.1] that viscosity solution of the problem (16), (17) is unique. Let $a>0$. Since the viscosity solution of the problem (1)-(8) is unique in the domains $G^{+} \backslash c l G_{+}^{K}$ and $G_{+}^{K+a}$, it is unique in the domain $G^{+}$.

\section{Acknowledgements}

The work was supported by Russian Foundation for Basic Research, project No. 20-01-00362.

\section{References}

Bardi, M. and Evans, L. C. (1984) On Hopf's formulas for solutions of Hamilton-Jacobi equations. Nonlinear Analysis: Theory, Methods \& Applications. vol. 8(11), pp. 1373-1381.

Bruckner, A. (1994). Differentiation of real functions. CRM monograph series, vol. 5, American Mathematical Society.

Crandall, M.G. and Lions, P.L. (1983) Viscosity solutions of Hamilton-Jacobi equations. Trans. Amer. Math. Soc. vol. 277(1), pp. 1-42.

Ferrera, J. (2014). An introduction to Nonsmooth Analysis. Elsevier/Academic Press, Amsterdam.

Filippova, T. (2013) State Estimation for a Class of Nonlinear Dynamic Systems through HJB Technique. $C y$ bernetic and Physics. vol. 2(3), pp. 127-132.

Kruger, A.Ya. (2003) On Fréchet Subdifferentials. $J$. Mathematical Sciences. vol. 116, pp. 3325-3358.

Kolpakova, E. and Tokmantsev, T. (2012) On Solution of Optimal Control Pproblems with Lipschitz Data. Cybernetic and Physics. vol. 1(4), pp. 266-273.

Saakian, D.B., Rozanova, O., Akmetzhanov, A. (2008) Dynamics of the Eigen and the Crow-Kimura models for molecular evolution. Physical Review E, 78, 041908.

Shagalova, L.G. (2021) A generalized solution of the Hamilton-Jacobi equation with a three-component Hamiltonian. Geometric Methods in Control Theory and Mathematical Physics. III International scientific conference. Abstracts of papers. Ryazan, Russia, April 26-30. p. 54. [in Russian].

Subbotin, A.I. (1995). Generalized Solutions of First Order PDEs: The Dynamical Optimization Perspective. Birkhauser, Boston.

Subbotina, N.N. (2006) The method of characteristics for Hamilton-Jacobi equations and applications to dynamical optimization. J. of Mathematical Sciences, vol. 135, no 3. Ser. Modern Math. Appl., vol. 20, pp. 2955-3091.

Subbotina, N.N., Shagalova, L.G. (2016) On the continuous extension of a generalized solution of the Hamilton-Jacobi equation by characteristics that form a central field of extremals. Proc. Steklov Inst. Math. (Suppl.), vol. 293, suppl. 1, pp. 183--198. 